\title{
Comparison of Smith-Purcell radiation models and criteria for their verification
}

\author{
D. V. Karlovets* and A. P. Potylitsyn \\ Applied Physics Department, Tomsk Polytechnic University, 634050, prospekt Lenina 30, Tomsk, Russia
}

(Received 20 June 2006; published 24 August 2006)

\begin{abstract}
The paper presents the analysis of the angular distributions of the incoherent Smith-Purcell radiation (SPR) generated by a charged particle passing over a periodic conducting structure (grating). The calculations were carried out for a grating which consists of conducting strips separated by vacuum gaps and a lamellar grating with the use of three models of this radiation: van den Berg's model, the surface current model, and the resonant diffraction radiation model. The two last models predict sufficiently close results for different geometries, while the results obtained with van den Berg's model predict primarily the SPR intensity which is by a few orders of magnitude lower for the electron energy $\approx 6 \mathrm{MeV}$. The authors propose simple criteria to verify the SPR models by comparing the SPR yields between the volume (with the thickness comparable with a grating period) and flat (with the thickness which is much less than that of a period) gratings; by comparing the azimuth dependencies of the SPR intensities from the flat gratings; by comparing the dependencies of the SPR intensity on the Lorentz factor of a particle.
\end{abstract}

DOI: 10.1103/PhysRevSTAB.9.080701

\section{INTRODUCTION}

In [1-4], the authors proposed using the coherent SmithPurcell radiation (SPR) of relativistic electrons to determine the length of short electron bunches (with the length less than $1 \mathrm{~mm}$ ). In recent experiments [5,6], the possibility of creating a monochromatic radiation source of the $\mathrm{THz}$ range on the basis of the SPR has been demonstrated. The authors of [7] proposed to use the SPR to construct a beam position monitor. In experiment [8], the possibility of applying the SPR as a spontaneous radiation mechanism for a free-electron laser has been investigated. However, until now no conventional model to calculate the characteristics of the SPR from relativistic electrons is available. For the nonrelativistic electron energies $\left(E_{e} \preceq 100 \mathrm{keV}\right)$, the approach developed by van den Berg $[9,10]$ ensures a reasonable agreement with experiments $[11,12]$.

For relativistic electrons along with van den Berg's model [13] alternative approaches have been developed, which are more descriptive and less cumbersome: a scalar diffraction model [14], an induced surface current model $[15,16]$, a resonant diffraction radiation model [17], a model based on the use of integral equations for an electric field of radiation [18].

However, the predictions of most of the models mentioned differ from van den Berg's model results by approximately 2 orders of magnitude for the electron with energy $\sim 20 \mathrm{MeV}$ [18] and by 6 orders of magnitude for the electron energy $E_{e}=855 \mathrm{MeV}$ [19].

The available experimental results do not provide an ultimate conclusion on the validity of one of these models. In experiment [20] carried out with the electron beam having the energy of $2.8 \mathrm{MeV}(\gamma=5.6)$, the measured level of the spontaneous radiation power exceeds van den

\footnotetext{
*Corresponding author: karlovets@tpu.ru
}

PACS numbers: 41.60.- m, 42.25.Fx, 42.79.Dj, 41.75.Ht

Berg's model predictions by more than 2 orders of magnitude. The authors of [4] came to the conclusion that the power of coherent SPR of electrons with the energy equal to $2.3 \mathrm{MeV}$ agrees satisfactorily with that predicted by the surface current model.

In one of the first experiments studying the SPR with the electron energy $E_{e}=3.6 \mathrm{MeV}$ [21], the authors obtained a good agreement with the calculation according to the current model, while the experimental results in [8] for $E_{e}=1.44 \mathrm{MeV}$ are in good agreement with van den Berg's model. Finally, one should point out that in experiment [19] the measured intensity of the SPR in the optical range for the electrons with energy $855 \mathrm{MeV}$ satisfactorily agrees with van den Berg's model and is by 6 orders of magnitude lower than the theoretical estimation of the surface current model.

A purposeful carrying out of an experiment to show the validity of one or the other of these models may be thought to be an important problem. However, the difficulties of absolute measurements of the SPR power within the demanded accuracy hamper performing such an experiment.

In this work the authors compare the calculated SPR characteristics of different models for characteristic grating types and it is shown that rejection of one or the other model may be achieved by using rather simple relative measurements.

\section{SMITH-PURCELL RADIATION MODELS BEING COMPARED}

\section{A. Van den Berg's model}

We will consider properties of SPR from gratings within the following types:

(1) "Volume" gratings: a lamellar grating [Fig. 1(a)] and a grating which consists of a periodic set of 


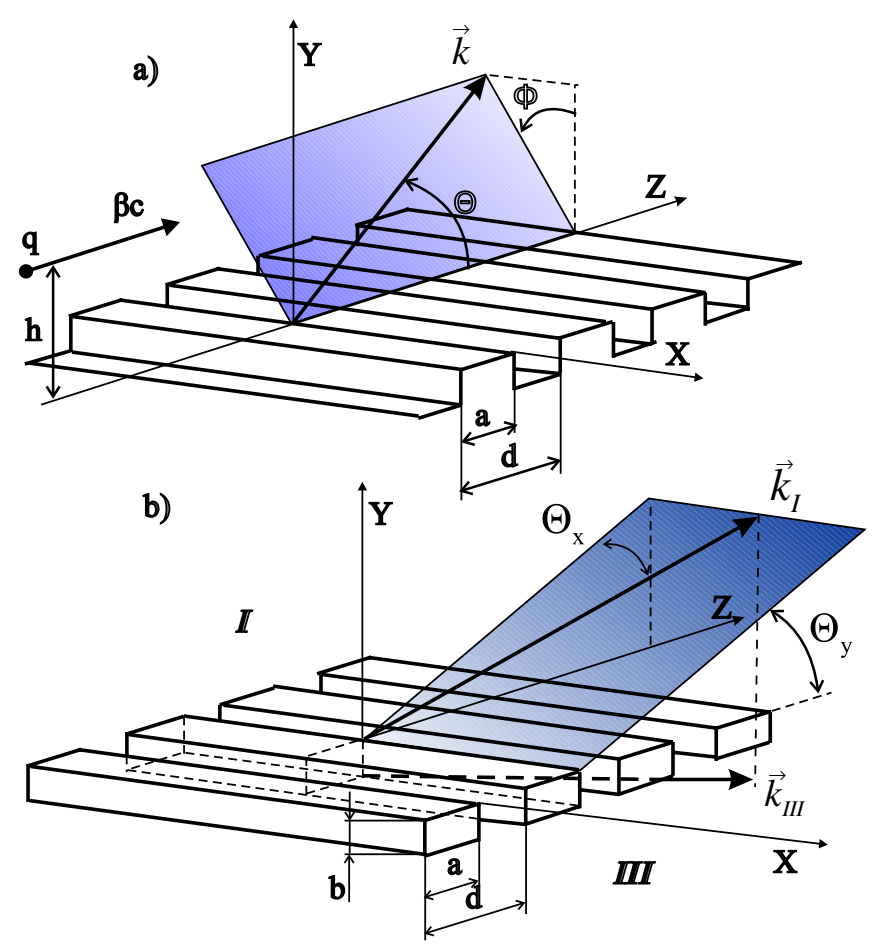

FIG. 1. (Color) Scheme of the Smith-Purcell radiation generation from the volume gratings: (a) lamellar grating; (b) grating with vacuum gaps.

conducting slabs separated by vacuum gaps [Fig. 1(b)].

(2) "Flat" gratings, which consist of perfect conducting infinitely thin strips oriented at $\Theta_{0}$ angle to the particle velocity and separated by vacuum gaps (Fig. 2).

From simple phase relations Smith and Purcell [22] derived a dispersion relationship for a fixed geometry to observe the monochromatic emission [see Fig. 1(a), where $\Theta, \Phi$ are polar and azimuth angles, $v=\beta c$ is the particle velocity, $d$ is the grating period] connecting the radiation wavelength and the observation angle $\Theta$ :

$$
\lambda_{n}=\frac{d}{n}\left(\frac{1}{\beta}-\cos \Theta\right)
$$

$n=1,2,3, \ldots$ is the diffraction order.

The so-called "projection" angles $\Theta_{x}$ and $\Theta_{y}$ are often used [Fig. 1(b)]: $\sin \Theta_{x}=\sin \Theta \sin \Phi, \sin \Theta_{y}=$ $\frac{\sin \Theta \cos \Phi}{\sqrt{1-\sin ^{2} \Theta \sin ^{2} \Phi}}$. In these variables,

$$
\lambda_{n}=\frac{d}{n}\left(\frac{1}{\beta}-\cos \Theta_{y} \cos \Theta_{x}\right) .
$$

In van den Berg's model the field of a moving charge is substituted for a packet of evanescent waves which are diffracting on the grating according to optics laws. Such a task was solved by the modal expansion technique in $[5,13,23]$. Denoting the impact parameter, i.e., the shortest



FIG. 2. Scheme of the Smith-Purcell radiation generation from the flat strip grating.

distance from a particle trajectory to the grating surface by $h$, the angular distribution of the SPR intensity per an electron per one grating period for $n$th diffraction order per unit of a solid angle can be written in the form [23]

$$
\begin{aligned}
\frac{d W_{n}}{d \Omega}= & \frac{\alpha \hbar c}{2 d} n^{2} \frac{\sin ^{2} \Theta \cos ^{2} \Phi}{\left(\frac{1}{\beta}-\cos \Theta\right)^{3}}\left|\Re_{n}\right|^{2} \\
& \times \exp \left[-\frac{h}{h_{\mathrm{eff}}} \sqrt{1+(\beta \gamma \sin \Theta \sin \Phi)^{2}}\right] .
\end{aligned}
$$

Here $\gamma$ is the Lorentz factor, $\alpha$ is the fine structure constant, $\hbar c=0.2 \mathrm{eV} \mu$ is the conversion constant, $h_{e f f}=\frac{\beta \gamma \lambda_{n}}{4 \pi}$ is the so-called effective parameter of electron coupling with a grating, $\left|\Re_{n}\right|^{2}$ is the radiation factor (the analog of the reflection factor in optics), playing the main role in the van den Berg's theory, which depends on the grating profile but not on the particle impact parameter.

The dependence of the radiation factor $\left|\Re_{n}\right|^{2}$ on different parameters was considered in detail in $[5,13]$ and others. There was shown in [5] that, for $\gamma=78$ and a lamellar grating [Fig. 1(a)], one can observe a periodic behavior of the radiation factor depending on a groove depth. The experimental results obtained in the cited work were in satisfactory agreement with theoretical calculations according to van den Berg's model.

This model in [23] was modernized for the grating with vacuum gaps [Fig. 1(b)]. For such a grating along with "reflected" wave which is radiated into the semispace above the grating where a particle passes (it is denoted by the symbol $I$ ), there will exist a "transmitted" wave which is radiated into the semispace denoted by a symbol $I I I$, as it was done in [23]. Its angular distribution of the SPR power is found by the expression similar to (3) where the "transmission factor" $\left|\mathfrak{I}_{n}\right|^{2}$ is used instead of the "radiation factor" $\left|\Re_{n}\right|^{2}$.

To find $\left|\Re_{n}\right|^{2}$ and $\left|\mathfrak{I}_{n}\right|^{2}$ it is necessary to know the coefficients of infinite Rayleigh series expansion for the electric and magnetic fields of a dispersed wave. If one cuts off the series at the $N$ th term (see the details in [23]), then in order to find the expansion coefficients one should solve the linear algebraic equations systems of $2 N+1$ (lamellar grating) and $4 N+2$ (the grating with vacuum gaps) 
dimensions. In [23] the dependence of the radiation factor on the parameters of the grating with vacuum gaps has been simulated for the ultrarelativistic case $(\gamma=1673)$ at $N=100$. It was shown that radiation factor $\left|\Re_{n}\right|^{2}$ can vary to a few orders of magnitude at small variations of the slab height and width. In our calculations we have chosen $N=$ 30 , which ensured a rather short computational time and enabled us to obtain the computational precision greater than 5 percent as compared with that when cutoff was made at the 100th term [23].

The system of algebraic equations for the magnetic field of a scattered wave totally defines the radiation factor (or transmission) in the plane perpendicular to the grating surface $(\Phi=0)$, because in this case the electric component of scattering wave vanishes at the expense of boundary condition imposed on the field strength functions. In the case of nonzero azimuth angle, we also solved the equation system of the similar dimension for the electric field strength.

\section{B. Surface current model}

In the surface current model [16,24], the SPR is generated by the current induced by the field of a particle moving in vacuum on a perfect conducting periodic surface. The expression for SPR intensity per an electron per one grating period according to the surface current (SC) model may be represented by analogy with (3):

$$
\begin{aligned}
\frac{d W_{n}}{d \Omega}= & \alpha \hbar c \frac{2 \pi}{d} \frac{n^{2}}{\left(\frac{1}{\beta}-\cos \Theta\right)^{3}}\left|\vec{R}_{n}\right|^{2} \\
& \times \exp \left[-\frac{h_{2}}{h_{\text {eff }}} \sqrt{1+(\beta \gamma \sin \Theta \sin \Phi)^{2}}\right],
\end{aligned}
$$

where $h_{2}$ is the impact parameter taken from the lower strip point (see Fig. 2). In the surface current model, the analog of the radiation factor is defined by the surface current on a grating unit cell:

$$
\left|\vec{R}_{n}\right|^{2}=|[\vec{n} \times[\vec{n} \times \vec{G}]]|^{2},
$$

where $\vec{n}=\{\sin \Theta \sin \Phi, \sin \Theta \cos \Phi, \cos \Theta\}$ is the unit vector in the direction of the SPR outgoing photon, and a complex vector $\vec{G}$ is expressed by the Fourier transform of the induced surface current density [16]. In the case when a grating period (a unit cell) consists of a finite number of flat strips oriented variously ("volume" grating), the vector $\vec{G}$ is written in the form of a vector sum across all the planes [24].

In the case when a unit cell consists of one strip only (with the width $a$ ) oriented at $\Theta_{0}$ angle to the particle velocity (see Fig. 2), the radiation factor is calculated according to the expression

$$
\begin{aligned}
\left|\vec{R}_{n}\right|^{2}= & \left(\sin ^{2} \Theta-2 \cos \Theta \sin \Theta \cos \Phi \tan \Theta_{0}+\tan ^{2} \Theta_{0}\left[1-\sin ^{2} \Theta \cos ^{2} \Phi+4\left(1-\sin ^{2} \Theta \sin ^{2} \Phi\right) \frac{(\beta \gamma \sin \Theta \sin \Phi)^{2}}{1+(\beta \gamma \sin \Theta \sin \Phi)^{2}}\right]\right) \\
& \times\left\{\left(\frac{\pi d \tan \Theta_{0}}{\beta \gamma \lambda_{n}} \sqrt{1+(\beta \gamma \sin \Theta \sin \Phi)^{2}}\right)^{2}+\left[\frac{\pi d}{\lambda_{n}}\left(\beta^{-1}-\cos \Theta-\sin \Theta \cos \Phi \tan \Theta_{0}\right)\right]^{2}\right\}^{-1} \\
& \times\left(\sinh ^{2} \chi+\sin ^{2} \frac{\Delta \varphi}{2}\right) \exp \left[\frac{a \sin \Theta_{0}}{2 h_{\text {eff }}} \sqrt{1+(\beta \gamma \sin \Theta \sin \Phi)^{2}}\right]
\end{aligned}
$$

where

$$
\begin{aligned}
\chi & =\frac{\pi a \sin \Theta_{0}}{\beta \gamma \lambda_{n}} \sqrt{1+(\beta \gamma \sin \Theta \sin \Phi)^{2}}, \\
\frac{\Delta \varphi}{2} & =\frac{\pi a \cos \Theta_{0}}{\lambda_{n}}\left(\beta^{-1}-\cos \Theta-\sin \Theta \cos \Phi \tan \Theta_{0}\right) .
\end{aligned}
$$

When a unit grating cell consists of two or more inclined strips, the expression for radiation factor becomes much more complicated. In this case one has to use computer simulation only.

\section{Resonant diffraction radiation model}

A comparatively simple model of the resonant diffraction radiation (RDR) enables one to obtain an analytical expression for the SPR intensity from a grating consisting of infinitely thin perfect conducting strips separated by vacuum gaps (see Fig. 2), if one knows the spectral-angular distribution of the diffraction radiation which is generated by a charged particle passing over an inclined conducting semiplane $\frac{d^{2} W_{D R}}{d \omega d \Omega}$ [17]. It is shown in the cited work that the RDR intensity may be given in the form of the product of three multipliers [which is in full analogy with a resonant transition radiation (RTR) [25]]:

$$
\frac{d^{2} W_{\mathrm{RDR}}}{d \omega d \Omega}=\frac{d^{2} W_{\mathrm{DR}}}{d \omega d \Omega} F_{n, \mathrm{cell}} F_{N} .
$$

Here $\frac{d^{2} W_{D R}}{d \omega d \Omega}$ is the intensity of diffraction radiation from a perfect conducting semiplane, $F_{n \text {,cell }}$ is the term taking into account the radiation interference of the $n$th order on a grating cell, and $F_{N}$ characterizes the interference from the grating consisting of $N$ cells.

The expression for intensity of the diffraction radiation from a semiplane was obtained on the basis of the exact solution of the Maxwell's equations by using the WienerHopf technique in [26]. It should be noted that in experiment [27] the measured characteristics of the optical dif- 
fraction radiation from the electrons with the Lorentzfactor $\gamma=2500$ are in good agreement with the calculations based on the perfect conducting semiplane model [28].

The applicability scope of the RDR model is determined by the inequality $\Theta_{\min } \leq \Theta \leq \Theta_{\max }$ (see Fig. 2).

By taking into account the phase shift $\Delta \varphi$ between waves radiated from the opposite edges of a strip, $F_{n \text {,cell }}$ is given in the form

$$
\begin{aligned}
F_{n, \text { cell }} & =\left|\exp \left(\chi-i \frac{\Delta \varphi}{2}\right)-\exp \left(-\chi+i \frac{\Delta \varphi}{2}\right)\right|^{2}= \\
& =4\left[\sinh ^{2} \chi+\sin ^{2}\left(\frac{\Delta \varphi}{2}\right)\right],
\end{aligned}
$$

where the values $\chi$ and $\frac{\Delta \varphi}{2}$ coincide with those from (7).
The interference factor for $N$ identical periods does not depend on the radiation mechanism (see e.g. [25]):

$$
F_{N}=\frac{\sin ^{2}\left(N \frac{\varphi_{0}}{2}\right)}{\sin ^{2}\left(\frac{\varphi_{0}}{2}\right)}, \quad \varphi_{0}=\frac{2 \pi d}{\lambda}\left(\frac{1}{\beta}-\cos \Theta\right) .
$$

In the case of a sufficiently large number of periods $N \gg 1$, the last expression is approximated by the $\delta$-function with a good accuracy:

$$
F_{N}=2 \pi N \delta\left(\varphi_{0}-2 k \pi\right), \quad k=1,2,3, \ldots
$$

The zeros of the $\delta$-function give the Smith-Purcell dispersion relation (1).

In such a way the expression for the angular distribution of Smith-Purcell radiation according to the RDR model in a unit solid angle per an electron per one period will be written:

$$
\begin{aligned}
\frac{d W_{n}}{d \Omega}= & \int \frac{d^{2} W}{d \omega d \Omega} F_{n, \text { cell }} F_{N} d \omega \\
= & \frac{\alpha \hbar c}{2 \pi} \frac{F_{n, \text { cell }}}{d\left(\beta^{-1}-\cos \Theta\right)^{2} \sqrt{1-(\sin \Theta \sin \Phi)^{2}}} \frac{1}{\left[\gamma^{-2}+(\beta \sin \Theta \sin \Phi)^{2}\right]\left[1-\beta\left(\cos \left[2 \Theta_{0}\right] \cos \Theta+\sin \left[2 \Theta_{0}\right] \sin \Theta \cos \Phi\right)\right]} \\
& \times\left[\left[\gamma^{-2}+(\beta \sin \Theta \sin \Phi)^{2}\right]\left[1+\beta \cos \Theta_{0} \sqrt{1-(\sin \Theta \sin \Phi)^{2}}\right]\left(1-\frac{\cos \Theta \cos \Theta_{0}+\sin \Theta \sin \Theta_{0} \cos \Phi}{\sqrt{1-(\sin \Theta \sin \Phi)^{2}}}\right)\right. \\
& \left.+(\sin \Theta \sin \Phi)^{2}\left[1-\beta \cos \Theta_{0} \sqrt{1-(\sin \Theta \sin \Phi)^{2}}\right]\left(1+\frac{\cos \Theta \cos \Theta_{0}+\sin \Theta \sin \Theta_{0} \cos \Phi}{\sqrt{1-(\sin \Theta \sin \Phi)^{2}}}\right)\right] \\
& \times \exp \left[-\frac{h_{1}}{h_{\text {eff }}} \sqrt{1+(\beta \gamma \sin \Theta \sin \Phi)^{2}}\right]
\end{aligned}
$$

Here $h_{1}$ is the impact parameter taken from the middle of the strip (see Fig. 2).

In the most interesting case $(\Phi=0)$, the formula obtained is essentially simplified:

$$
\begin{aligned}
\left.\frac{d W_{n}}{d \Omega}\right|_{\Phi=0}= & \alpha \hbar c \frac{2}{\pi} \frac{1}{d\left(\beta^{-1}-\cos \Theta\right)^{2}} \frac{\left(\beta^{-1}+\cos \Theta_{0}\right)\left(1-\cos \left[\Theta-\Theta_{0}\right]\right)}{\beta^{-1}-\cos \left[\Theta-2 \Theta_{0}\right]} \exp \left[-\frac{h_{1}}{h_{\text {eff }}}\right] \\
& \times\left\{\sinh ^{2}\left[\frac{\pi a \sin \Theta_{0}}{\lambda_{n} \beta \gamma}\right]+\sin ^{2}\left[\frac{\pi a}{\lambda_{n}}\left(\frac{\cos \Theta_{0}}{\beta}-\cos \left(\Theta-\Theta_{0}\right)\right)\right]\right\} .
\end{aligned}
$$

The last addend in the braces attains its maximum value (equal to 1) provided the following condition is fulfilled:

$$
\frac{\sin [\Theta / 2]}{\sin \left[\Theta / 2-\Theta_{0}\right]}=\left(k+\frac{1}{2}\right) \frac{d}{a n}, \quad k=0,1,2,3, \ldots
$$

from which the coupling between the radiation angle $\Theta$ and the blazing angle $\Theta_{0}$ for the maximum value of $F_{n \text {,cell }}$ may be obtained. It should be noted that formula (14) was obtained for the relativistic case $(\gamma \gg 1, \beta \approx 1)$.

Note also that, for a flat grating consisting of infinitely thin strips $\left(\Theta_{0}=0\right)$, the SPR in regions $I$ (above the grating) and III (below the grating) have identical properties. In fact, 


$$
\begin{aligned}
\left.\frac{d W_{n}}{d \Omega}\right|_{\Theta_{0}=0}= & \frac{2 \alpha \hbar c}{\pi} \frac{\sin ^{2}\left[\frac{\pi a}{\lambda_{n}}\left(\beta^{-1}-\cos \Theta\right)\right]}{d\left(\beta^{-1}-\cos \Theta\right)^{3} \sqrt{1-(\sin \Theta \sin \Phi)^{2}}}\left[\left[\beta^{-1}+\sqrt{1-(\sin \Theta \sin \Phi)^{2}}\right]\left(1-\frac{\cos \Theta}{\sqrt{1-(\sin \Theta \sin \Phi)^{2}}}\right)\right. \\
& +\frac{(\sin \Theta \sin \Phi)^{2}}{\left[\gamma^{-2}+(\beta \sin \Theta \sin \Phi)^{2}\right]}\left[\beta^{-1}-\sqrt{1-(\sin \Theta \sin \Phi)^{2}}\right]\left(1+\frac{\cos \Theta}{\left.\left.\sqrt{1-(\sin \Theta \sin \Phi)^{2}}\right)\right]}\right. \\
& \times \exp \left[-\frac{h_{1}}{h_{\mathrm{eff}}} \sqrt{1+(\beta \gamma \sin \Theta \sin \Phi)^{2}}\right]
\end{aligned}
$$

It is not difficult to see that this expression is symmetrical with respect to substitution of $\Phi \rightarrow \Phi \pm \pi$.

Unlike the model in [16], where the surface current was determined as induced by a "nondistorted" field of a passing particle, which coincided with the field in vacuum, in the RDR model an exact solution of the Maxwell's equations [26] was used which takes into account the particle field distortion caused by a conducting surface of a strip.

\section{Comparison of models for thin strips grating}

Let us compare the analytical expressions for the SPR intensity according to the surface current model and the resonant diffraction radiation model. To obtain the expression for $\left|\vec{R}_{n}\right|^{2}$ for the simplest case, when $\Phi=0$, for formula (4) one can write

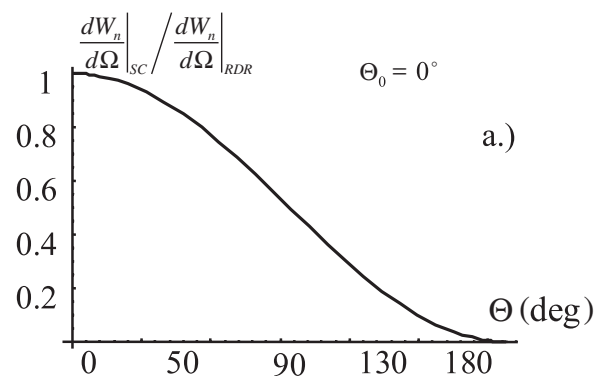

$$
\begin{aligned}
\left.\frac{d W_{n}}{d \Omega}\right|_{\mathrm{SC}}= & \alpha \hbar c \frac{2}{\pi} \frac{1}{d\left(\beta^{-1}-\cos \Theta\right)^{2}} \frac{\sin ^{2}\left[\Theta-\Theta_{0}\right]}{\beta^{-1}-\cos \left[\Theta-2 \Theta_{0}\right]} \\
& \times\left(\sinh ^{2} \chi+\sin ^{2} \frac{\Delta \varphi}{2}\right) \exp \left[-\frac{h_{2}-\frac{a}{2} \sin \Theta_{0}}{h_{\text {eff }}}\right],
\end{aligned}
$$

As it follows from Fig. 2 for such gratings, the relationship $h_{1}=h_{2}-\frac{a}{2} \sin \Theta_{0}$ is fulfilled; then by taking into account (13) one can obtain the ratio between the SPR intensities for both models:

$$
\left.\frac{d W_{n}}{d \Omega}\right|_{\mathrm{SC}} /\left.\frac{d W_{n}}{d \Omega}\right|_{\mathrm{RDR}}=\frac{1+\cos \left[\Theta-\Theta_{0}\right]}{\left(\beta^{-1}+\cos \Theta_{0}\right)},
$$

So, for $\beta \rightarrow 1$ and strip inclinations $\Theta_{0}=0$ and $\Theta_{0}=$ $26^{\circ}$, the dependence of ratio (17) on the polar angle is

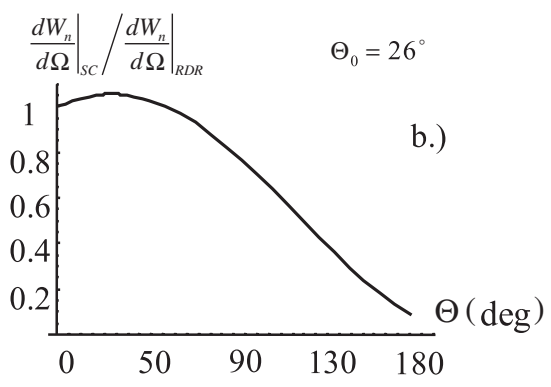

FIG. 3. Ratio between the SPR intensities for the surface current and RDR models in the relativistic case $(\beta \approx 1)$.
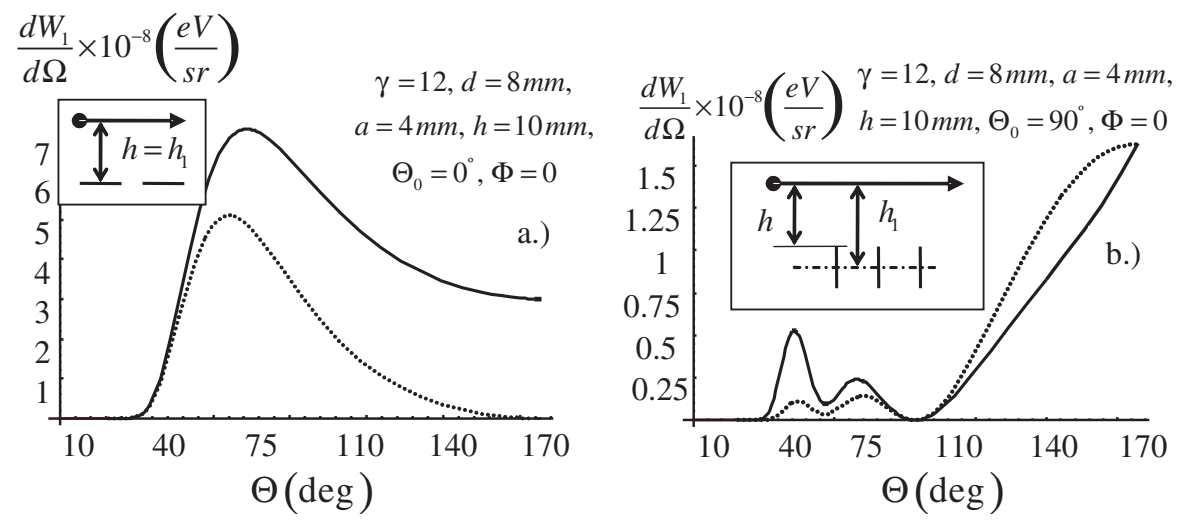

FIG. 4. Angular distribution of the SPR intensity according to the surface current model (a dotted line) and the RDR model (a solid line). 

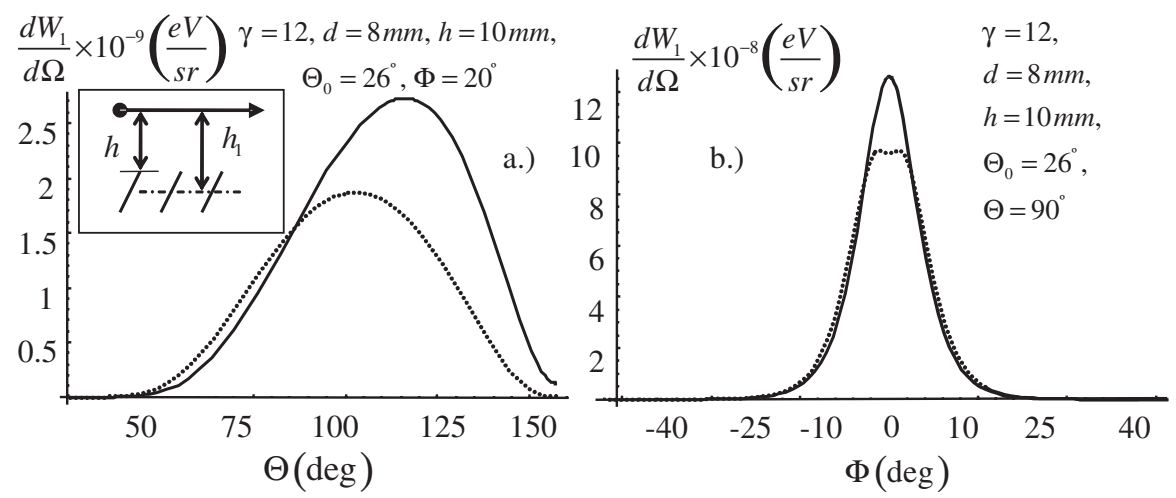

FIG. 5. Angular distribution of the SPR intensity according to the surface current model (a dotted curve) and the RDR model (solid line).

given in Fig. 3. Comparing the SPR intensity from gratings with variously oriented strips for the RDR model (a solid curve) and the current model (a dot curve) for the case $d=$ $a \cos \Theta_{0}$ (see Figs. 4 and 5), one can find that these models predict practically identical behavior of the SPR intensity in the region of polar angles $\Theta \leqslant \pi / 4$.

As it follows from Figs. 4(a) and 4(b), the SPR yield from a flat grating (see the inset) considerably exceeds the radiation yield from the "perpendicular" strip grating.

In the relativistic case the SPR is "concentrated" within a region of small azimuth angles as for large azimuth angles the SPR intensity is suppressed exponentially. In the region of $\Phi \preceq(\gamma \sin \Theta)^{-1}$, the difference in the intensity values is not substantial (see Figs. 4 and 5).

It should be noted that in the case of $\Phi=0, \Theta_{0}=0$ formulas (3), (4), and (12) are of similar structure, since $h=h_{1}$. Really,

$$
\begin{aligned}
\left.\frac{d W_{n}}{d \Omega}\right|_{\mathrm{vdB}}= & \frac{\alpha \hbar c}{2 d} \frac{\sin ^{2} \Theta}{\left(\beta^{-1}-\cos \Theta\right)^{3}} n^{2}\left|\Re_{n}\right|^{2} \exp \left[-\frac{h}{h_{\mathrm{eff}}}\right], \\
\left.\frac{d W_{n}}{d \Omega}\right|_{\mathrm{SC}}= & \alpha \hbar c \frac{2}{d \pi} \frac{\sin ^{2} \Theta}{\left(\beta^{-1}-\cos \Theta\right)^{3}} \\
& \times \sin ^{2}\left[\frac{\pi a}{\lambda_{n}}\left(\beta^{-1}-\cos \Theta\right)\right] \exp \left[-\frac{h}{h_{\mathrm{eff}}}\right], \\
\left.\frac{d W_{n}}{d \Omega}\right|_{\mathrm{RDR}}= & \alpha \hbar c \frac{2}{d \pi} \frac{\left(\beta^{-1}+1\right)(1-\cos \Theta)}{\left(\beta^{-1}-\cos \Theta\right)^{3}} \\
& \times \sin ^{2}\left[\frac{\pi a}{\lambda_{n}}\left(\beta^{-1}-\cos \Theta\right)\right] \exp \left[-\frac{h}{h_{\mathrm{eff}}}\right]
\end{aligned}
$$

As one can see from the above expressions, the main difference in the predicted SPR intensity between the current model (including the RDR model) and the one of van den Berg is due to behavior of the radiation factor $\left|\Re_{n}\right|^{2}$. In the current model and in that of the resonant diffraction radiation, the dependence of the SPR intensity upon the diffraction order $n$ appears only via the wavelength $\lambda_{n}$. The analog of the value $n^{2}\left|\Re_{n}\right|^{2}$ in the surface current model and the RDR model does not practically depend on $\gamma$ in the relativistic case [see Eqs. (6) and (12)]. In van den Berg's model the dependence on the Lorentz factor is included into the radiation factor, which results in substantial differences in the dependence of the SPR intensity on a particle energy (see details below).

Finally, the values $\left|\Re_{n}\right|^{2}$ in van den Berg's model, $\left|\vec{R}_{n}\right|^{2}$ in the surface current model, and $F_{n \text {,cell }}$ in the resonant diffraction radiation model depend only on the ratios between grating parameters $b / d, a / d$ and do not depend on the values of the latter [23].

\section{CALCULATED CHARACTERISTICS OF SMITH-PURCELL RADIATION}

\section{A. Comparison of SPR intensity from different type structures}

Let us compare the angular distribution of the calculated SPR intensity from different gratings according to van den Berg's model, the surface current model, and the resonant diffraction radiation model. The angular dependencies of the SPR intensity from a grating with vacuum gaps for the following parameters $(\gamma=12, d=8 \mathrm{~mm}, \quad a=d / 2$, $\Phi=0$ ) are presented in Fig. 6. As it follows from expressions (3), (4), and (12), with fulfilling the condition $h / h_{\text {eff }} \gtrsim 1$ for large azimuth angles the SPR intensity will be exponentially suppressed, that is why the impact parameter $h=10 \mathrm{~mm}$ was chosen for calculations.

The intensity of SPR from a flat grating according to the RDR model is shown by a solid line, the intensity in region $I$ calculated according to van den Berg's model for a volume grating $(b / d=1 / 8)$ and a flat "thin" grating $(b / d=0.001, b \ll \lambda)$ are shown by dotted and dashdotted lines, respectively.

First of all, it should be noted that the difference in intensities of radiation from a grating whose thickness is less than the SPR wavelength according to both models exceeds 4 orders of magnitude. Further, van den Berg's model predicts a higher radiation yield from a volume grating than from a flat one. 


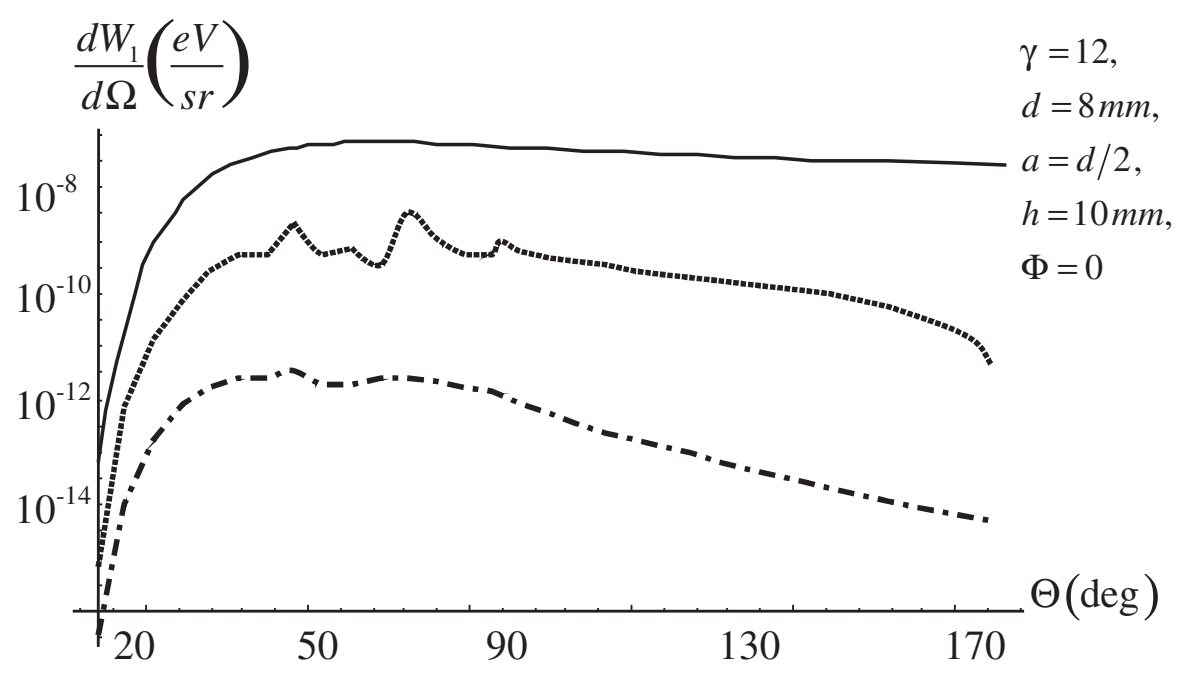

FIG. 6. The angular distribution of the SPR intensity for a thin grating with vacuum gaps: a solid line for the RDR model; a dotted line $(b / d=1 / 8)$ and a dash-dotted line $(b / d=0.001)$ for van den Berg's model.

Similar dependencies for a lamellar grating are presented in Fig. 7: a solid line shows the intensity according to the surface current model $(b / d=1 / 8)$, dotted and dashdotted lines demonstrate the intensities according to van den Berg's model $(b / d=1 / 8$ and $b / d=0.001)$. As it occurred in the previous case, the surface current model predicts much higher radiation intensity for most of the observation angles.

It should be noted that the SPR intensity according to van den Berg's model for "almost flat" gratings $(b / d=$ 0.001) with vacuum gaps as well as for the "lamellar grating" type differ from each other insignificantly (see the dash-dotted curves in Figs. 6 and 7), while drawing the analogy to the resonant transition radiation one should expect that for the grating with vacuum gaps the value $n^{2}\left|\Re_{n}\right|^{2}$ (the analog of $F_{n \text {,cell }}$ factor) has to exceed sufficiently the analogous value for the lamellar grating. In fact, the RTR output will be the higher, the greater will be the difference (in quantity) of dielectric constants of the two layers making up the structure period. If one of the layers is the vacuum one, then the RTR intensity reaches the maximum possible value. For the lamellar grating with $b / d=$ 0.001 the periodic structure is close to a uniform plane for which $F_{n \text {,cell }}=0$, i.e., no radiation is generated.

Figures 8 and 9 give the comparison of the angular dependence of the SPR intensity for a volume grating



FIG. 7. Angular distribution of the SPR intensity for the lamellar grating: according to the current model - a solid line $(b / d=1 / 8)$; according to van den Berg's model - a dotted line $(b / d=1 / 8)$ and a dash-dotted line $(b / d=0.001)$. 


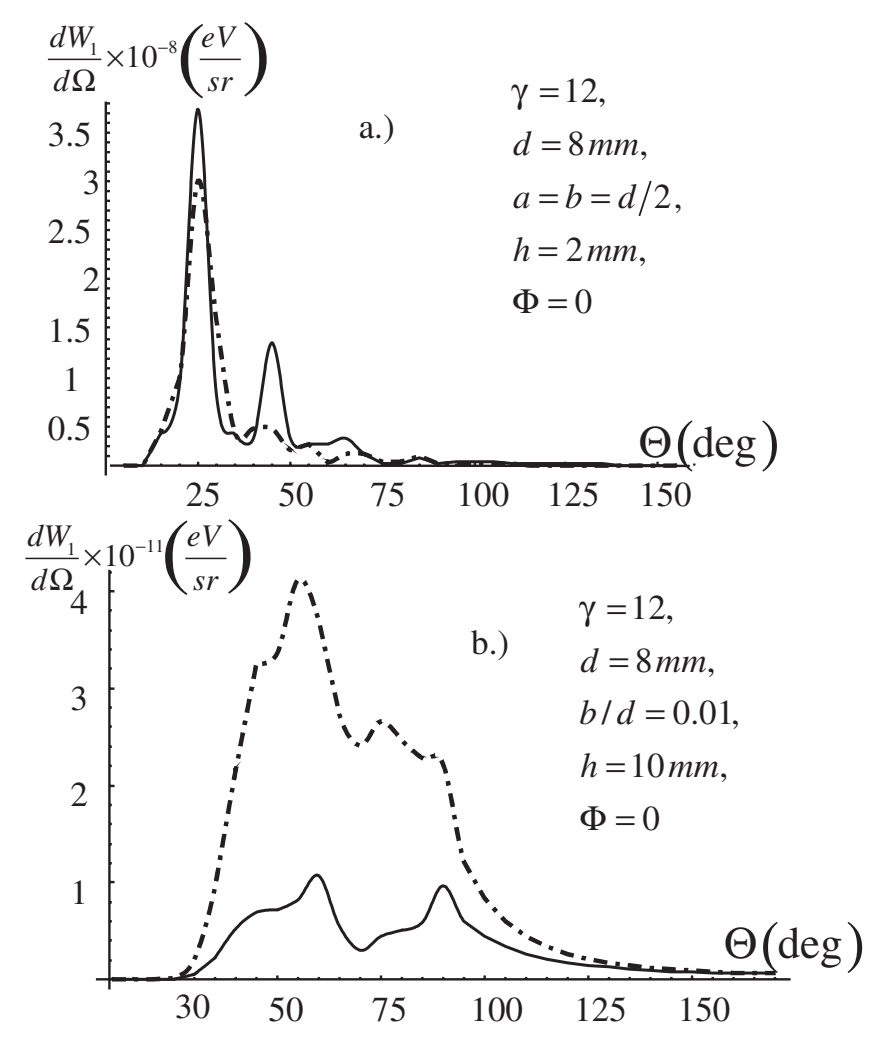

FIG. 8. Angular distribution of the SPR intensity for a grating with vacuum gaps according to van den Berg's model (a solid line - in region $I$; a dash-dotted line - in region $I I I$ ): (a) volume grating $(a=b=d / 2)$; (b) flat grating $(b / d=0.01)$.

with vacuum gaps and strips $4 \mathrm{~mm}$ thick, a flat grating with $b / d=0.001$, and perpendicular grating with $a / d=0.001$. The dash-dotted line denotes the intensity in region $I I I$, the curve according to the RDR model is shown by a dotted line. As the other conditions being equal, the intensity of the SPR from a "thick" grating is by 2 orders of magnitude greater.

As it follows from Fig. 9, for a flat grating the RDR model gives the result which is approximately 2000 times higher than that of van den Berg's model, and for the perpendicular grating [see Fig. 9(b)] this excess differs by 100 times. Another difference of van den Berg's model from the surface current and RDR ones is the fact that the predicted intensity from the vertical strip grating is higher than that from the flat grating [see Figs. 9(a) and 9(b)] while the situation is opposite for the current model (see Fig. 4).

Finally, it can be shown that according to van den Berg's model the SPR intensity below the grating (in region $I I I$ ) may be several times higher than in region $I$ [see Figs. 8(a) and $8($ b)] for small thickness gratings with vacuum gaps $(b \ll d)$ which is also in agreement with the calculations of [23]. This difference vanishes with the strip height approximate to zero [29].



FIG. 9. Angular distribution of the SPR intensity (a) for a flat grating with thin strips: according to van den Berg's model (a solid line - in region $I$; a dash-dotted line-in region $I I I$; a dotted line - for RDR model, should be multiplied by indicated factors); (b) the same for a perpendicular grating.

\section{B. Azimuth intensity distribution of SPR}

As it has already been shown [30] in the case when a particle passes at a distance larger than $h_{\text {eff }}$ from a grating, the azimuth distribution of the SPR intensity, as a rule, is of a single-maximum character with a maximum in the plane normal to the grating. In case when $h \ll h_{\text {eff }}$, a different situation is observed for volume gratings.

We have calculated the azimuth distribution of the SPR intensity with the use of all three models: van den Berg's, the surface current, and the resonant diffraction radiation models for the impact parameter equal to $1 \mathrm{~mm}$ and $\gamma=$ 12 (see Figs. 10 and 11). Normalization was applied for direct comparison of azimuth distributions. For instance, the SPR intensity calculated according to the current model is by more than 4 orders of magnitude higher than the calculation results obtained according to van den Berg's model (see Fig. 10). In Fig. 10 the curves are presented according to van den Berg's model for thick gratings $(b \sim$ $\lambda$ ), namely, for the volume grating with vacuum gaps $4 \mathrm{~mm}$ high (a solid curve) and for a lamellar grating $4 \mathrm{~mm}$ high (a dot curve). A dash-dotted curve depicts the intensity for a rectangular profile according to the current model for the same parameters. It can be seen that a more or less pro- 

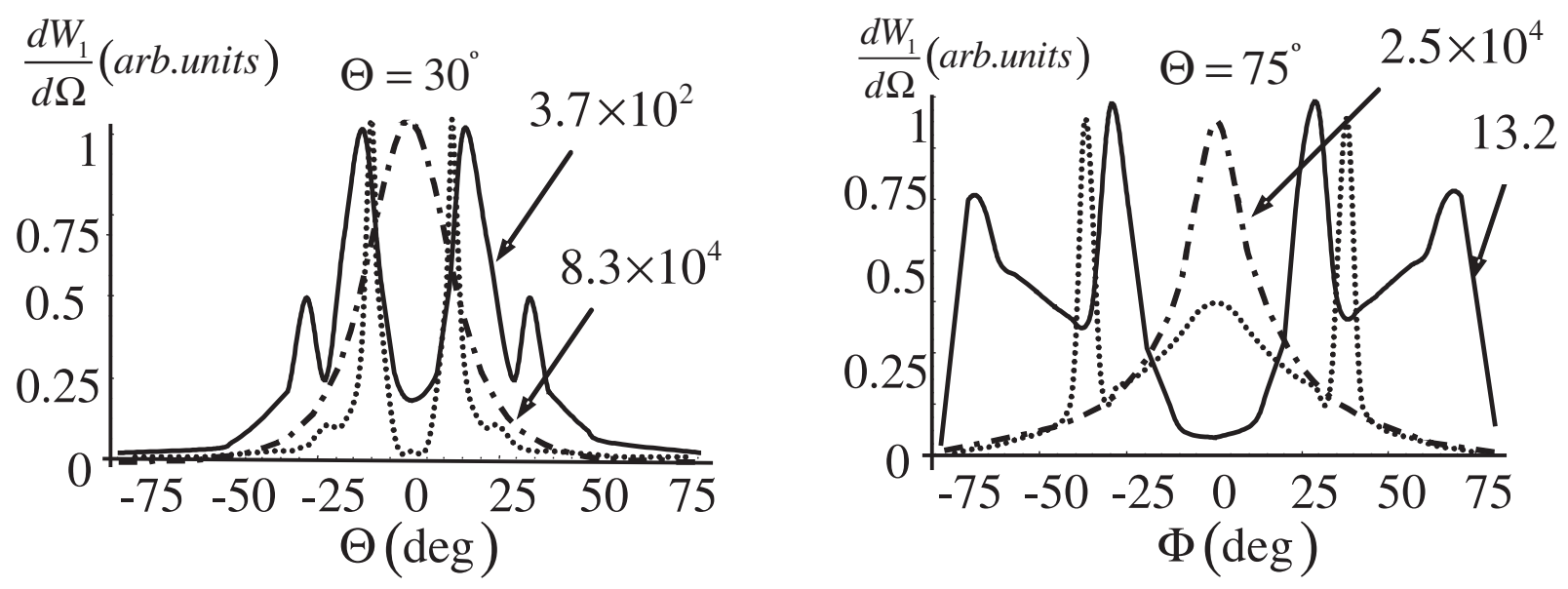

FIG. 10. Azimuth distribution of the SPR intensity according to van den Berg's model ( $\gamma=12, d=8 \mathrm{~mm}, a=d / 2, h=1 \mathrm{~mm}$, $b_{\text {vol gr }}=b_{\text {lam gr }}=4 \mathrm{~mm}$; a dotted line - lamellar grating; solid line - grating with vacuum gaps), according to SC model ( $\gamma=12$, $d=8 \mathrm{~mm}, a=d / 2, h=1 \mathrm{~mm}, b_{\text {lam gr }}=4 \mathrm{~mm}$; a dash-dotted line-lamellar grating).

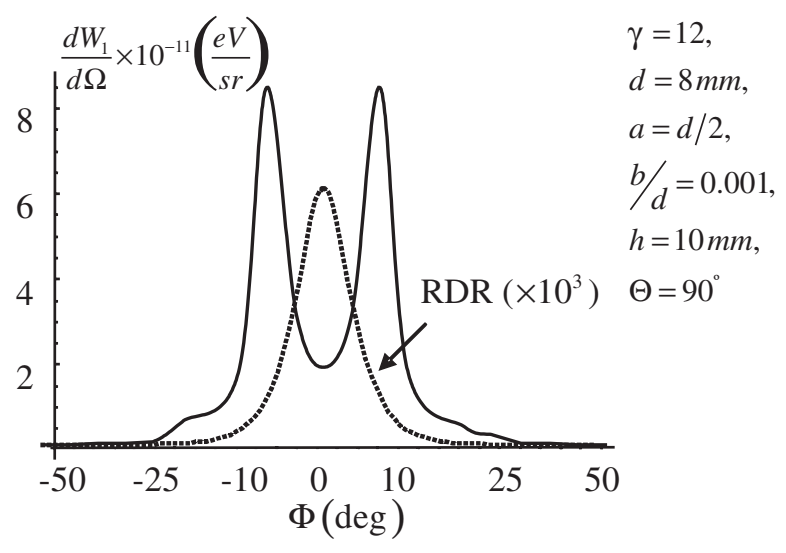

FIG. 11. Azimuth dependence of the SPR intensity for a flat grating according to van den Berg's model (a solid line) and according to the RDR model (a dotted line, should be multiplied by $\left.10^{3}\right)$.

nounced lobe-shaped distribution is observed for different polar angles $\Theta$, however, the intensity distribution is not always characterized by a minimum in the vicinity of $\Phi=$ 0 . So, the current model for these parameters predicts a maximum in this plane. However, one can show that according to the current model while reducing the width of the strip $a$ the azimuth dependence also becomes lobe shaped (already at $a=d / 4$ ).

A much greater difference among the SPR models in the predicted azimuth distributions of intensity is observed for the case $b \ll \lambda$. Figure 11 presents a corresponding dependence for the grating with vacuum gaps and $b / d=$ 0.001 and the impact parameter equal to $10 \mathrm{~mm}$ according to van den Berg's model. Such a dependence with a minimum in the zero azimuth plane is observed for small impact parameters $(h<\lambda)$ as well, however, it is not observed for a flat grating according to the current and RDR models, where, as it has been mentioned, there is a single-maximum azimuth distribution for all $\Theta$.

\section{Dependence on Lorentz factor}

According to van den Berg's model, the increase of particle energy does not lead to the rise of the SPR yield $[13,23]$, at least in the polar angle region, ensuring the necessary accuracy of calculations (usually $\Theta>30^{\circ}$ ). At the same time according to the surface current model an opposite behavior is observed. So, in Figs. 12 and 13 a comparison of the SPR intensity is depicted for the both models for the grating with vacuum gaps. The calculation was done for fixed observation angles $\Theta=120^{\circ}, \Phi=0$. The difference is seen to be due to the form of the energy loss dependence on the Lorentz factor: van den Berg's model predicts the decrease in the SPR output with the energy growth [13], while the current model predicts the increase [30].

\section{CONCLUSION AND DISCUSSION}

The comparison of different SPR models analyzed in the previous sections enables one to come to the following conclusions:

(1) There exists a difference in the SPR intensity between the volume and flat gratings: the SPR yield from the volume grating predicted by van den Berg's model for a moderately relativistic case is by 2 orders of magnitude higher practically for all the polar angles;

(2) There exists a difference in the azimuth dependencies for the flat gratings: the current and RDR models predict a single-maximum angular distribution with maximum in the plane perpendicular to the 

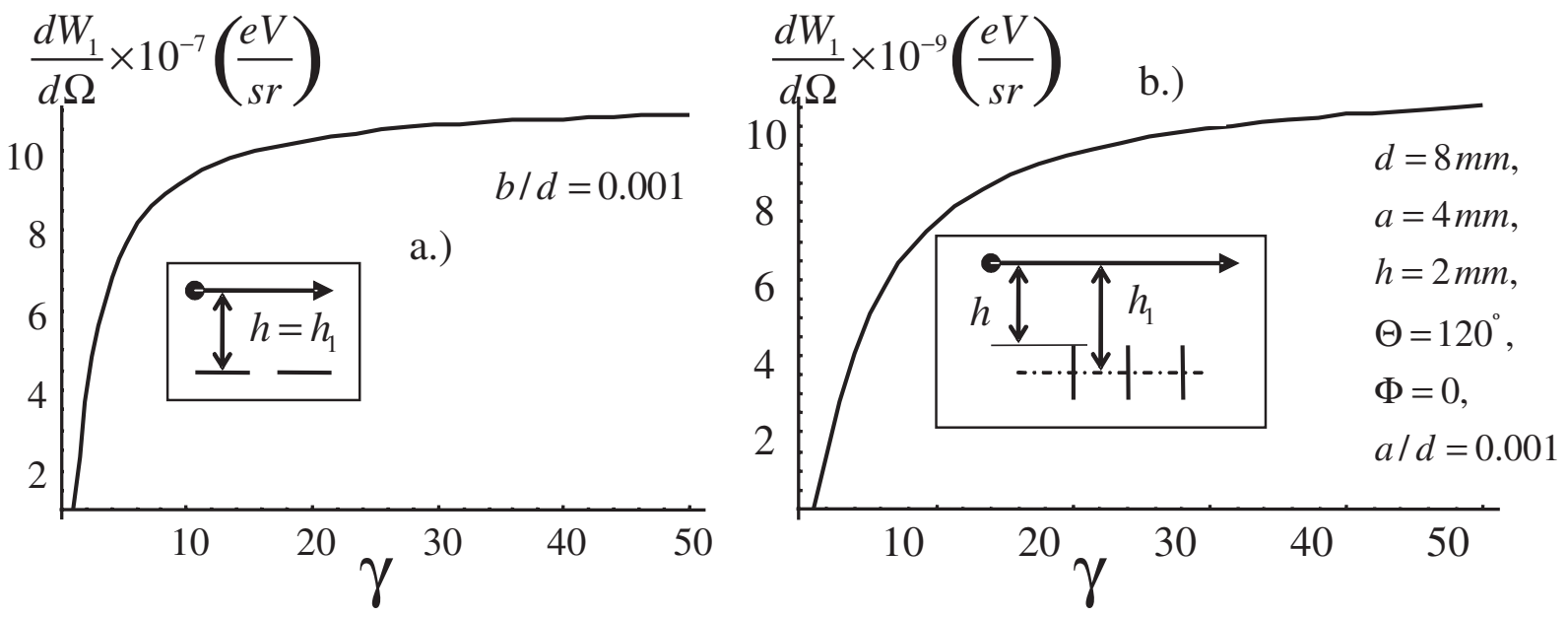

FIG. 12. Dependence of the SPR intensity on the Lorentz factor according to the RDR model: (a) for a flat grating; (b) for a vertical strip grating.
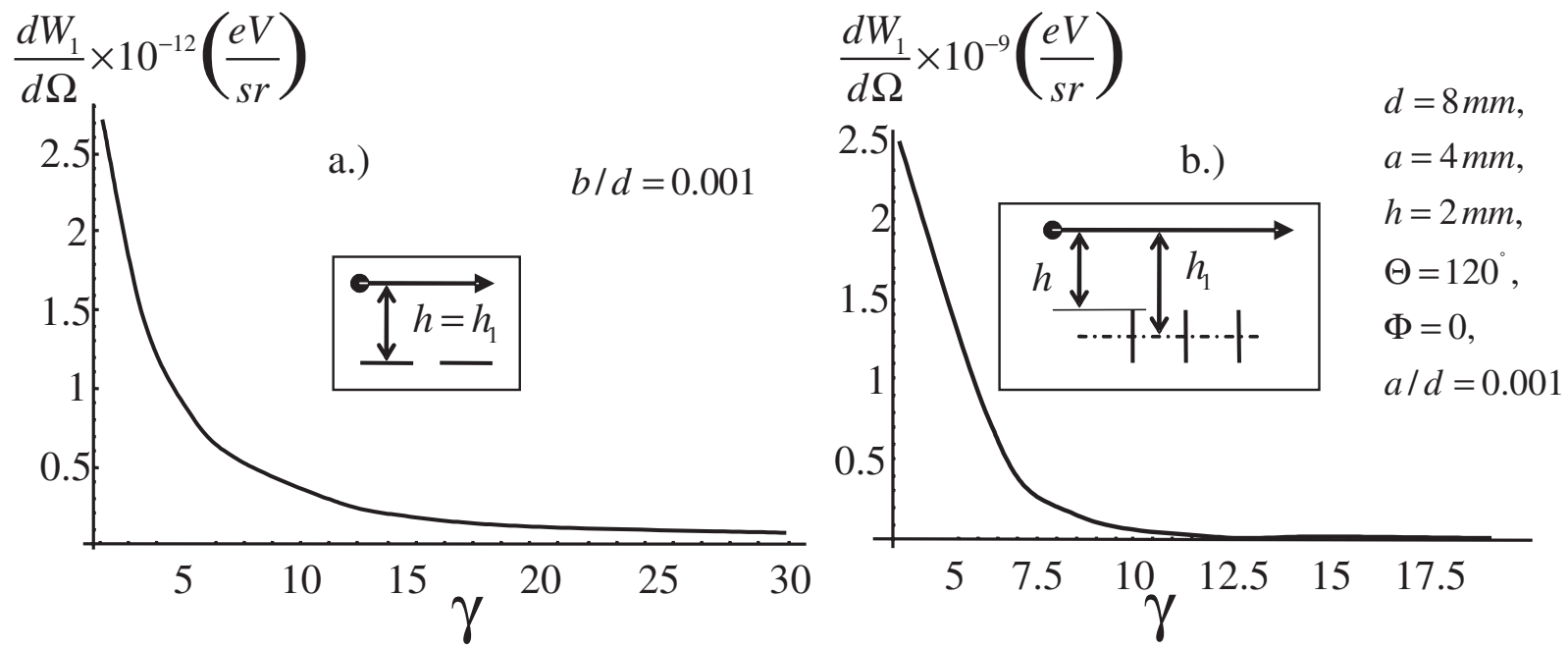

FIG. 13. Dependence of the SPR intensity on the Lorentz factor according to van den Berg's model: (a) for a flat grating; (b) for a vertical strip grating.

grating $(\Phi=0)$, while van den Berg's model predicts the distribution with a minimum in the plane $\Phi=0$;

(3) There exists a difference in the SPR yield dependence on the Lorentz factor of a particle: van den Berg's model predicts the yield decrease with the energy growth, while the current and RDR models predict the yield increase.

In van den Berg's model the electric field of a particle is substituted for a set of plane waves incident on the grating. The right-hand side of the Maxwell's equations in this model contains only the density of the charged particle current, while the induced surface current density is not taken into account. This drawback might be allowed for by modifying this model, which was proposed in [31].

Even in comparing the models qualitatively, one may note that the surface current and RDR models assume fewer approximations whose character of affecting the final result is not obvious. These models not only ensure short computation time as compared with that of van den Berg's model, they are more illustrative as it was pointed out in $[16,17]$.

The verification of validity of these models is supposed to be a highly urgent task and it can be carried out on experiments with moderately relativistic electrons (5$10 \mathrm{MeV}$ energy) of a linear accelerator or a microtron with a bunch duration $\tau_{b} \sim 10^{-11} \mathrm{sec}$. In this case, the SPR intensity in the region of wavelengths $\lambda \gtrsim c \tau_{b}=$ $3 \mathrm{~mm}$ grows due to coherent effects by $\sim N_{e}$ times $\left(N_{e}\right.$ is the number of electrons per a bunch). As was pointed out in [5], the characteristics of the coherent SPR are uniquely determined by those of a single charge radiation. For a bunch with $N_{e} \approx 10^{9}$ electrons, a detector with an aperture of $\approx 10^{-3} \mathrm{sr}$ will detect the power $\sim 1 \mathrm{~mW}$ in passing a 


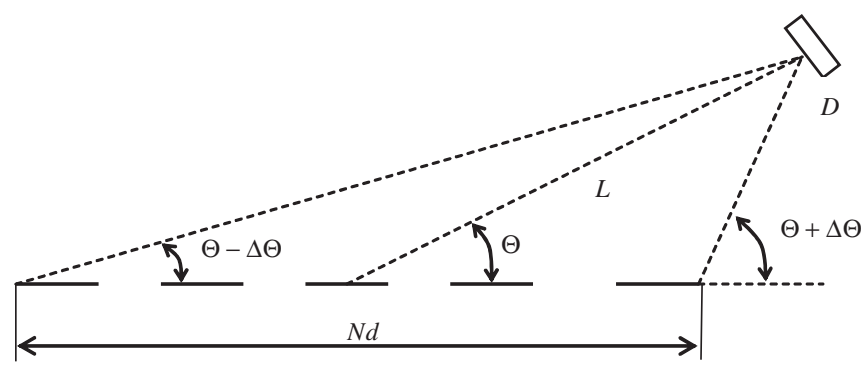

FIG. 14. A scheme of the detector disposition with respect to the grating.

10-psec bunch, if the SPR intensity is equal to $\sim 10^{-10} \mathrm{eV} / \mathrm{sr}$ and, respectively, $\sim 100 \mathrm{~mW}$ for the SPR intensity $\sim 10^{-8} \mathrm{eV} / \mathrm{sr}$ (see Figs. 4-9). In this wavelength range (which corresponds to the frequency band $\sim 100 \mathrm{GHz}$ ) the modern detectors operating at room temperature may ensure a required sensitivity [32].

We would like to emphasize that the conclusions and calculations of the models discussed in this paper are valid to place a detector in the so-called "far" zone only, i.e., in neglecting the grating length $N d$ as compared to the distance $L$ between the grating and a detector. The characteristics of the transition radiation and diffraction radiation in the "prewave" zone, i.e., in geometry where the above approximation is not fulfilled, have been considered in $[33,34]$. We can give a rather approximate criterion of a prewave zone for the SPR starting from the following concepts.

For a grating with $N$ periods, the RDR model [see formula (10)] gives the following equation for the monochromaticity of the SPR lines (for a rather small detector aperture):

$$
\frac{\Delta \lambda_{n}}{\lambda_{n}} \approx \frac{1}{N}
$$

By applying dispersion relation (1), one can obtain from (19) the formula coupling the variation of the polar angle $\Delta \Theta$ in the process of emitting waves from the initial and final grating period for the same monochromaticity:

$$
\frac{\Delta \lambda_{n}}{\lambda_{n}} \approx \frac{\sin \Theta}{1-\cos \Theta} \Delta \Theta \approx \frac{1}{N} .
$$

Then we have

$$
\Delta \Theta \approx \frac{1}{N} \frac{1-\cos \Theta}{\sin \Theta}=\frac{1}{N} \tan \frac{\Theta}{2} .
$$

From the elementary scheme of a detector position with respect to the grating (see Fig. 14), one can obtain the formula to change the polar angle $\Delta \Theta$ of the wave vector:

$$
\Delta \Theta \approx \frac{N d \sin \Theta}{2 L} .
$$

By comparing the obtained formula with (21) we receive the inequality:

$$
\frac{N d \sin \Theta}{2 L} \ll \frac{\tan \Theta / 2}{N}
$$

or

$$
L \gg N^{2} d \frac{1+\cos \Theta}{2} .
$$

One can easily see that in the region of $\Theta>\pi / 2$ angles the far-zone criterion can be readily satisfied.

It is clear that carrying out absolute measurements of the coherent SPR power is connected with a rather complicated experimental problem. However, as it has been shown in the previous paragraphs, the comparison of the relative dependencies (such as azimuth and energy ones) of the coherent SPR yield from gratings of comparatively simple profile will allow one to confirm or reject the SPR models considered.

\section{ACKNOWLEDGMENTS}

The authors are grateful to Dr. G. Kube and Dr. P. Karataev for stimulating discussions. The work was partly supported by the grant of Ministry of Education and Science of the Russian Federation "Advancement of the Scientific Potential of High Education", No. 2.1.1.889.

[1] M. C. Lampel, in Advanced Accelerator Concepts: Eighth Workshop, edited by W. Lawson, C. Bellamy, and D. Brosius (AIP, New York, 1999), pp. 785-794.

[2] D. C. Nguyen, Nucl. Instrum. Methods Phys. Res., Sect. A 393, 514 (1997).

[3] S.E. Korbly, W. J. Brown, M. A. Shapiro, and R. J. Temkin, in Proceedings of the Particle Accelerator Conference, 2001, Chicago, USA (IEEE, New York, 2001), pp. 2347-2349.

[4] A. Doria, G. P. Gallerano, E. Giovenale, G. Messina, G. Doucas, M.F. Kimmitt, H.L. Andrews, and J.H. Brownell, Nucl. Instrum. Methods Phys. Res., Sect. A 483, 263 (2002).

[5] Yukio Shibata, Shigeru Hasebe, Kimihiro Ishi, Shuichi Ono, Mikihiko Ikezawa, Toshiharu Nakazato, Masayuki Oyamada, Shigekazu Urasawa, Toshiharu Takahashi, Tomochika Matsuyama, Katsuhei Kobayashi, and Yoshiaki Fujita, Phys. Rev. E 57, 1061 (1998).

[6] S. E. Korbly, A. S. Kesar, J. R. Sirigiri, and R. J. Temkin, Phys. Rev. Lett. 94, 054803 (2005).

[7] G. Doucas, M. F. Kimmit, J. H. Brownell, S. R. Trotz, and J.E. Walsh, Nucl. Instrum. Methods Phys. Res., Sect. A 474, 10 (2001).

[8] H. Backe, W. Lauth, H. Mannweiler, H. Rochholz, K. Aulenbacher, R. Barday, E. Euteneuer, K.-H. Kaiser, G. Kube, F. Schwellnus, and V. Tioukine, in NATO Workshop "Advanced Radiation Sources and Applications" (Springer, New York, 2006), pp. 267-282.

[9] P. M. van den Berg, J. Opt. Soc. Am. 63, 1588 (1973).

[10] P. M. van den Berg, J. Opt. Soc. Am. 63, 689 (1973). 
[11] A. Gover, P. Dvorkis, and U. Elisha, J. Opt. Soc. Am. B 1, 723 (1984).

[12] Y. N. Adischev, A. V. Vukolov, D. V. Karlovets, A. P. Potylitsyn, and G. Kube, Pis'ma Zh. Eksp. Teor. Fiz. 82, 192 (2005) [JETP Lett. 82, 174 (2005)].

[13] O. Haeberle, Ph.D. thesis, University Louis Pasteur, France, 1994.

[14] B. M. Bolotovski and G. V. Voskresenskii, Sov. Phys. Usp. 11, 143 (1968).

[15] J. Walsh, K. Woods, and S. Yeager, Nucl. Instrum. Methods Phys. Res., Sect. A 341, 277 (1994).

[16] J. H. Brownell, J. Walsh, and G. Doucas, Phys. Rev. E 57, 1075 (1998).

[17] A.P. Potylitsyn, Nucl. Instrum. Methods Phys. Res., Sect. B 145, 60 (1998).

[18] A. S. Kesar, Phys. Rev. ST Accel. Beams 8, 072801 (2005).

[19] G. Kube, H. Backe, H. Euteneuer, A. Grendel, F. Hagenbuck, H. Hartmann, K. H. Kaiser, W. Lauth, H. Schöpe, G. Wagner, and Th. Walcher, Phys. Rev. E 65, 056501 (2002).

[20] K. J. Woods, J.E. Walsh, R. E. Stoner, H. G. Kirk, and R. C. Fernow, Phys. Rev. Lett. 74, 3808 (1995).

[21] J. H. Brownell, G. Doucas, M.F. Kimmitt, J. H. Mulvey, M. Omori, and J. E. Walsh, J. Phys. D 30, 2478 (1997).
[22] S. J. Smith and E. M. Purcell, Phys. Rev. 92, 1069 (1953).

[23] G. Kube, Nucl. Instrum. Methods Phys. Res., Sect. B 227, 180 (2005).

[24] J.H. Brownell and G. Doucas, Phys. Rev. ST Accel. Beams 8, 091301 (2005).

[25] X. Artru, G. B. Yodh, and G. Mennessier, Phys. Rev. D 12, 1289 (1975).

[26] A. P. Kazantsev and G. I. Surdutovich, Dokl. Akad. Nauk USSR 147, 74 (1963) [Sov. Phys. Dokl. 7, 990 (1963)].

[27] T. Muto, S. Araki, R. Hamatsu, H. Hayano, T. Hirose, P. Karataev, G. Naumenko, A. Potylitsyn, and J. Urakawa, Phys. Rev. Lett. 90, 104801 (2003).

[28] A. P. Potylitsyn, Nucl. Instrum. Methods Phys. Res., Sect. B 145, 169 (1998).

[29] G. Kube (private communication).

[30] A. P. Potylitsyn, Phys. Lett. A 238, 112 (1998).

[31] V. Kumar and K.-J. Kim, Phys. Rev. E 73, 026501 (2006).

[32] B. N. Kalinin, G. A. Naumenko, A. P. Potylitsyn, G. A. Saruev, L. G. Sukhikh, and V. A. Cha, Pis'ma Zh. Eksp. Teor. Fiz. 84, 136 (2006) [JETP Lett. 84, 3 (2006)].

[33] V. A. Verzilov, Phys. Lett. A 273, 135 (2000).

[34] A.P. Potylitsyn, in NATO Workshop "Advanced Radiation Sources and Applications", Ref. [8], pp. 149163. 